"bends"; comprising limb pains (which were not dangerous) or manifestations in the cardiovascular, respiratory, or central nervous system (which could endanger life). The chronic decompression syndrome of caisson disease of bone could result in crippling arthritis, and could occur even after properly performed decompression and in the absence of any previous manifestations of acute decompression sickness. It was presumed that caisson disease was due to "silent bubbles," which obstructed blood vessels in bone. In order to detect such silent bubbles (and thus to give warning that further compression followed by slow decompression was required) an ultrasonic transducer device had been used. Discussing experimental studies of this, Professor Walder described results obtained after this apparatus had been clipped on to the ear of a guinea-pig. The presence of minute bubbles in the ear tissues had been found to interfere with the display pattern on the oscilloscope and hence gave a a warning of their presence. Guinea-pigs exposed to compression were monitored during subsequent decompression by this means; recompression was carried out if bubbles were detected. All animals monitored in this way survived, whereas control animals died; at necropsy the former were free from bubbles in the blood stream and tissues, but in the latter bubbles were present in both.

Dr. M. H. Surofp (New York) described the application of hyperbaric oxygen therapy in the treatment of raised intracranial pressure. This had been investigated in dogs by the inflation of a balloon placed intracranially within the extradural compartment, which resulted in immediate hemiplegia and coma. The same procedure carried out in animals receiving $100 \%$ oxygen at three times atmospheric pressure produced evidence of temporary discomfort only but no evidence of neurological defect. In subsequent chronic experiments there was $100 \%$ mortality in the treated animals in contrast to a $50 \%$ mortality in dogs treated in the hyperbaric oxygen chamber two or three times daily. To date, six patients in coma with high intracranial pressure had been treated with hyperbaric oxygen, all with immediate improvement. In one case the cerebrospinal-fluid pressure measured by spinal manometry fell by more than half, and in another the tense flap of a cranial decompression operation became soft. It was postulated that the beneficial effects of the hyperbaric oxygenation were produced by a combination of improved oxygenation of cerebral tissue coupled with cerebral vasoconstriction, which broke the vicious circle of cerebral oedema- anoxia-increased oedema. Concluding, Dr. Sukoff suggested that possibly patients with high intracranial pressure requiring urgent neurosurgery might benefit if the operation were to be performed in a hyperbaric chamber.

\section{Large-bowel Obstruction Post Partum}

Dr. E. B. Tover (Toronto) gave an account of a recently described syndrome of postpartum large-bowel obstruction produced by the involuting uterus becoming jammed into the brim of the pelvis, and thereby obstructing the sigmoid colon as this crossed the pelvic brim. Typically the onset was within 24 to 48 hours following delivery, and there were the typical features of intestinal obstruction. The pelvic examination revealed the uterus to be tightly wedged within the pelvis, while radiologically there was considerable distension of the bowel down to the pelvic brim, where there was a sudden cut-off in the gas shadow. If recognized at once the condition was treated by placing the patient in the knee-chest position and pushing the uterus out of the pelvis by gentle pressure. Some late cases had progressed to volvulus and even perforation. Of 20 cases no fewer than 16 had followed caesarean section.

\title{
Tissue and Organ Transplantation
}

\section{[From a Spectal Correspondent]}

The College of Pathologists held their first symposium on 23 and 24 February in London on the subject of "Tissue and Organ Transplantation."

Dr. J. R. Batchelor (London) discussed transplantation antigens and their genetic background, pointing out that, though there were 30 years of work in animals to guide the work in man that was now in progress, some elucidation of the systems of compatibility was still necessary. To find out whether human white blood cells carried histocompatibility antigens a series of human volunteers had been paired and skin grafts exchanged between them until an antibody response was detected. Both leucocyte-agglutinating and lymphocyte-toxic antibodies had been found, and Dr. Batchelor suggested that the corresponding isoantigens were H antigens. Quantitative absorption analysis had so far led to the identification of 25 antigens of varying immunogenic strength. Population genetic studies had shown that there was a restricted number of immunogenetic systems, while the results of family studies indicated that some antigens were segregated from each other and that some were transmitted to the offspring in groups.

\section{Nature of Graft Rejection}

Discussing the role of lymphocytes in graft rejection, Dr. J. L. TURK (London) said that during the experimental production of humoral antibodies the lymph nodes drain- ing the site of the injection of antigen showed hyperplasia of the lymphoid follicles. Germinal centres appeared and many plasma cells were seen at the corticomedullary junction. In delayed hypersensitivity an area of the cortex-known as the paracortical area, or the thymus-dependent area-was the scene of proliferation of large pyroninophilic cells. These so-called immunoblasts then divided either into more immunoblasts or into small lymphocytes, and their number was maximal at the fifth day after stimulation. After the administration of antilymphocytic serum the paracortical area became filled with proliferating fibroblasts, and most immunosuppressive agents had been found to delay immunoblast production. The antigen was probably conveyed to the draining lymph nodes by lymphocytes, and hence one might expect to see changes in lymphocytes in the region of grafts. This was the case, for lysosomes were seen in $80 \%$ of such lymphocytes instead of the usual $10 \%$

Dr. D. C. Dumonde (London) considered the role of the macrophage in transplantation immunity. Macrophages were seen in rejected skin homografts, but their origins were not clear. In vitro, macrophages had been found to ingest antibody-coated cells, to take part in cell-contact reactions, and to help in the immunological reactions of the lymphocytes. It was still undecided whether macrophages could be produced by conversion of lymphocytes, or even whether they produced antibodies.
Dr. G. MölLER (Stockholm) pointed out that the homograft reaction was a very useful tool for studying the genetic complexities of tissue antigens. The serum antibodies had the capacity to kill some but not all target cells-and at least two molecules of antibody were required to bind the complement molecule, which actually penetrated the cell membrane to kill the cell. Both IgM and IgG antibodies could kill cells, though the latter could become resistant to antibodies and grow even better until rejected in the usual way. Host antibodies could also react with the antigens of the donor cells without killing the cells, and homograft rejection thus be avoided. Continuing, Dr. Möller said that cell-mediated immunity was less well defined. Nevertheless, if sensitized lymphocytes were dropped on to cells in tissue culture cytotoxicity was found to occur after contact for 24 to 48 hours. The effect was increased by adding phytohaemagglutinin or streptolysin to the cells.

\section{Modification of Host Response}

Dr. N. A. Mitchison (London) described the mechanism of induction of immunological tolerance. This was a central phenomenon, affecting the sensitization of the small lymphocyte itself, and the target for all immunosuppressive therapy. In the adult the administration of large quantities of antigen was known to produce immunological 
paralysis, but a surprising finding had been that in certain circumstances small doses of antigen had the same effect-for example, after irradiation or immunosuppressive drugs. Giving small doses of cells was similar to giving protein antigens, and one possible way of inducing tolerance in the future was to identify the latter and to produce them synthetically.

To explain the immunological deficiencies of thymectomized mice Dr. Delphine Parrot (London) described the histology of the lymph nodes draining areas of immunological stimulation. She called attention to the appearances of the postcapillary venuleswhich were collapsed and hard to find in thymectomized animals but prominent with plump endothelial cells in normal ones. The thymus-dependent area of the lymph node was depleted in neonatal thymectomized animals, but repopulation of the area by small lymphocytes occurred three to four weeks after a thymus graft had been implanted. Radioactively labelled cells could be observed to emerge from the thymus graft and migrate to the thymus-dependent area. Continuing, Dr. Parrott pointed out that after oxozalone had been applied to the skin blast-cell proliferation occurred in the draining lymph nodes in normal animals. In neonatal thymectomized mice, on the other hand, no response occurred. Similar findings were obtained using skin grafts; such grafts were not rejected by the neonatal thymectomized animals-but the late response of the lymph nodes, in which germinal centres and plasma cells appeared, still occurred. The exact relationship of the thymus to the lymph nodes was still not clear and there might also be humoral factors concerned in the transformation of lymphocytes into immunoblasts.

\section{Immunosuppression}

Professor M. F. A. Woodruff (Edinburgh) gave an account of the immunological properties of antilymphocytic serum. It had long been known that this could be prepared by injecting lymphocytes into another species, and that it caused a profound lymphopenia in the original animal about four hours after injection-and subsequently the number of lymphocytes in the peripheral blood gradually rose. In the initial work on antilymphocytic serum very little prolongation of homograft survival had been achieved and no destruction of lymphocytes had occurred. Nevertheless, with the more potent sera developed by using thoracic-duct lymphocytes as the antigen the survival of renal homografts had been prolonged. Drainage of the thoracic duct, irradiation before administration, and giving corticosteroid drugs were found to enhance the effect of antilymphocytic serum, but adrenalectomy and irradiation after administration opposed it. The antibody appeared to consist entirely of IgG immunoglobulin, but fractions of this had been prepared and were under investigation.

Dr. M. C. Berendaum (London) outlined the great improvement in the results of kidney transplantation in man. In 1965 only $6 \%$ of patients died in the three months following transplantation, and an increasing number of transplants were functioning for a year or more. Deaths were now associated mainly with the rejection crisis-at which time there was both an increasing need for immuno- suppressive drugs, together with a decreased tolerance to the drugs. These features were not yet explained. Immunosuppressive drugs had many side-effects, including impairment of wound healing, of antibody formation, of phagocytosis, of inflammatory response, and of the activity of serum complement, as well as leucopenia and a probable reduction in interferon production. In future the better selection of kidney donors should allow the use of smaller doses of immunosuppressive drugs.

Dr. J. F. Mowbray (London) said that it had been found that a plasma-protein fraction called fraction $C$ had been found to inhibit the rejection of allografts of rat skin. Further experiments in mice, using the haemagglutination response to sheep red cells, had shown that a single dose of this fraction completely suppressed this response. Though this immunosuppression was short-lived, two periods of immunosuppression occurred-the first 16 hours and the second 96 hours after injection-after which the animal was found to be tolerant to further injections of sheep red cells. More recently a highly purified form of ribonuclease had been derived from fraction C, which Dr. Mowbray was satisfied was the cause of the immunosuppressive effect.

\section{Leucocyte Antigens}

The analysis of leucocyte antigens with the help of a computer was described by Dr. J. J. VAN ROod (Leiden, Netherlands). Sera from 60 pregnant patients had been typed against 100 random people. The analysis revealed two groups of antibodies and their alleles. Family studies on 40 families had shown that some combinations were relatively common. Theoretical mating studies showed that parents and sibling could be identical, compatible, or incompatible with respect to leucocyte antigens, but that siblings would have identical antigens three times more often than parents and their children. Leucocyte antigens appeared to have a definite role in the acceptance or rejection of grafts, and this was supported by the correlation that had been found between leucocyte compatibility and the histological appearances of biopsies of kidney grafts taken at least one year after transplantation. Thus the incidence of rejection was considerably less than expected where the leucocyte antigens between donor and recipient were identical, about the expected figure when the antigens were merely compatible, and more than expected where the antigens were incompatible. When the donor was a sibling and the leucocyte antigens were incompatible the rejection rate was about $75 \%$. When the donor was a parent and the antigen incompatible, however, the rate was only $50 \%$. Further analysis revealed that in all the parent-child grafts that survived the incompatibility was confined to one antigen, and that the remaining antigens were identical.

\section{Organ Transplants}

Despite the administration of immunosuppressive drugs most patients with kidney transplants, Professor K. A. Porter (London) said, had at least one rejection episode. This might occur within an hour of transplanta- tion, when it was usually due either to presensitization of the recipient or to major blood group incompatibility. Acute rejection in the first week was well recognized clinically, and histologically there was massive round-cell infiltration and oedema, with damage to the tubules and venules-though the arterioles and glomeruli remained intact. Experimentally the first change was damage to peritubular capillaries by pyroninophilic cells. Similar changes to those described earlier by Dr. Turk were also seen in the paracortical areas of draining lymph nodes. Clinically, rejection after the first week had the same features, but histologically there was the additional feature of fibrinoid necrosis of the arteriolar walls-which was found even in patients without a rise in blood pressure. This fibrinoid material had been shown to be IgM globulin by immunofluorescent techniques. Electron microscopy also showed platelet clumping in the glomerular capillaries, and it had been found that this could be reversed by giving prednisone. When there was insidious late rejection the histological appearances resembled membranous glomerulonephritis, with diffuse or lobular thickening of capillary walls; the material responsible for this also appeared to be IgM globulin. Histologically these four forms of rejection had to be differentiated from fat embolism, and from the development of genuine glomerulonephritis in the transplanted kidney, while the clinical manifestations were indistinguishable.

\section{Cartilage Transplantation}

Discussing cartilage transplantations, Dr. T. GiBson (Glasgow) said that allogeneic transplants produced no immunological response. Studies with radioactive sulphur had proved that the donor chondrocytes survived. Grafts of whole cells freed by enzyme treatment from the matrix still elicited no response, and none occurred even when the recipient was presensitized with a skin graft. Unfortunately, homografts of cartilage were limited in their use by the difficulty of separating the cartilage from other tissues which were rejected-though one possibility was to use them for grafts to the head of the femur.

Dr. J. F. Loutit (Harwell) said that grafts of haemopoietic tissue were really seeds rather than true grafts. Much work had been done on the morphological identification of stem cells, which at present could be recognized only because they produced colonies after transplantation to animals that had been totally irradiated. Colonies could be produced by transplants of bone marrow, foetal liver, juvenile spleen, and-to a lesser extent -adult spleen and circulating white cells. Studies in mice suggested that there was one stem cell per 1,000 marrow cells or per 100,000 whole blood leucocytes. These stem cells were probably not reticulum cells or small lymphocytes derived from the lymph nodes or thymus, though possibly they were small lymphocytes derived from the bone marrow.

Dr. J. Chalmers (Edinburgh) said that both autografts and homografts of bone behaved in the same way. The success of a graft was not dependent on its viability, because the graft itself died anyway and merely acted as a scaffold for new host bone. 